\title{
Dental and periodontal phenotypes of Dlx2 overexpression in mice
}

\author{
JIEWEN DAI* , JIAWEN SI ${ }^{*}$, NINGJUAN OUYANG* , JIANFEI ZHANG, \\ DANDAN WU, XUDONG WANG and GUOFANG SHEN
}

\begin{abstract}
Department of Oral and Cranio-maxillofacial Surgery, Shanghai Ninth People's Hospital, Shanghai Jiao Tong University School of Medicine, Shanghai Key Laboratory of Stomatology, Shanghai 200011, P.R. China
\end{abstract}

Received November 23, 2015; Accepted December 12, 2016

DOI: $10.3892 / \mathrm{mmr} .2017 .6315$

\begin{abstract}
Distal-less homeobox 2 (Dlx2) is a member of the homeodomainfamily of transcription factorsandis importantfor the development of cranial neural crest cells (CNCCs)-derived craniofacial tissues. Previous studies revealed that Dlx2 was expressed in the cementum and a targeted null mutation disrupted tooth development in mice. However, whether Dlx 2 overexpression may impair in vivo tooth morphogenesis remains to be elucidated. The present study used a transgenic mouse model to specifically overexpress Dlx2 in neural crest cells in order to identify the dental phenotypes in mice by observation, micro-computed tomography and histological examination. The Dlx2-overexpressed mice exhibited tooth abnormalities including incisor cross-bite, shortened tooth roots, increased cementum deposition, periodontal ligament disorganization and osteoporotic alveolar bone. Therefore, Dlx 2 overexpression may alter the alveolar bone, cementum and periodontal ligament (PDL) phenotypes in mice.
\end{abstract}

\section{Introduction}

Epithelial-mesenchymal interactions are important for tooth development and various molecules, including bone morphogenetic protein 4 (Bmp4), lymphoid enhancer binding factor 1 , Wnt family member $10 \mathrm{~A}$, distal-less homeobox 2 (D1x2), Dlx5 and msh homeobox 1 (Msx1) are expressed during these processes and have been demonstrated to be important components of the tooth initiation and odontogenic patterning signaling pathways (1-6). Dlx2 is a member of the vertebrate

Correspondence to: Dr Guofang Shen or Dr Xudong Wang, Department of Oral and Cranio-maxillofacial Surgery, Shanghai Ninth People's Hospital, Shanghai Jiao Tong University School of Medicine, Shanghai Key Laboratory of Stomatology, 639 Zhizaoju Road, Shanghai 200011, P.R. China

E-mail: maxillofacsurg@163.com

E-mail: xudongwang70@hotmail.com

*Contributed equally

Key words: tooth root, distal-less homeobox 2, overexpression, cementum, alveolar bone
Dlx gene family, which is composed of six members organized into three convergent pairs of genes on chromosome 2 in mice (7,8). Dlx1 and Dlx2 are expressed in the epithelium and the cranial neural crest cell (CNCC)-derived mesenchyme of the mandibular and maxillary processes, and these genes are important for the development of craniofacial skeleton tissues (9-11). Previous studies revealed that D1x2-null and overexpressing mutants exhibited malformation of craniofacial tissues (11-16). Thomas et al (17) reported that the development of maxillary molars required regional specification of a population of CNCCs by the Dlx1 and Dlx 2 homeobox genes, and newborn mice with null mutations in the Dlx1 and Dlx2 genes had no maxillary molars; however, all other teeth were present (17). This phenotype may be due to a defect in the mesenchyme whereby odontogenic cells are reprogrammed to become chondrogenic, resulting in the replacement of maxillary molar teeth with ectopic cartilage. An 'odontogenic homeobox code' for dentition patterning based on the spatially restricted expression of homeobox genes in the first branchial arch mesenchyme was previously proposed. It was also proposed that the Dlx1 and Dlx 2 genes were specifically involved in the pattern of molar tooth development $(17)$. Lézot et al $(18,19)$ also reported that Dlx2 expression was evident in the molar and incisor root epithelia during initial root formation and may constitute a landmark for cementoblast subpopulations of epithelial origin involved in root morphogenesis and cementogenesis $(18,19)$. A previous study revealed that the deletion or mutation of Dlx3 may lead to major dentin defects through changes in the regulation of dentin sialophosphoprotein (20).

However, it is clear from previous D1x2-knockout studies that Dlx 2 may contribute to tooth development and whether Dlx 2 overexpression may influence the in vivo phenotypes of dental structures in mammals remains to be elucidated. The present study used a transgenic mouse overexpressing D1x2 in neural crest cells (NCCs) to determine how Dlx2 overexpression influences dental and periodontal tissues in mice. This analysis revealed that the mice exhibited tooth abnormalities, including incisor cross-bite, shortened tooth roots, increased cementum deposition, periodontal ligament (PDL) disorganization and osteoporotic alveolar bone.

\section{Materials and methods}

Mouse strains. Wnt1-Cre transgenic mice were obtained from the Jackson Laboratory for Genomic Medicine (Farmington, 
CT, USA). Previous studies used Wnt1-Cre mice crossed with Rosa R26R reporter mice to indicate precisely where and when the Cre recombinase was active during tooth development, including condensed dental mesenchyme, dental papilla, dental pulp, odontoblasts, dentine matrix, cementum and PDL, and used these mice to investigate the functions of genes in CNCCs during tooth development $(2,21)$. Transgenic mice conditionally overexpressing Dlx2 (iZEG-Dlx2) were constructed as described in our previous study (13). Wnt1-Cre transgenic mice were mated with iZEG-Dlx2 transgenic mice to obtain double transgenic offspring (Wnt1Cre::iZEG-Dlx2) that specifically overexpressed Dlx 2 in tissues derived from NCCs and the mice were genotyped with polymerase chain reaction (PCR) using primers to detect Cre recombinase (Cre) and enhanced green fluorescent protein (EGFP), as described in our previous study (13). Mice (including male and female mice) from a C57BL/6J genetic background were used in the current study and non-recombined littermates were used as controls. All mice were housed in a specific pathogen-free laboratory animal room at a temperature of $22^{\circ} \mathrm{C}$. The light cycle consisted of $12 \mathrm{~h}$ light and $12 \mathrm{~h}$ dark. The animal experimental procedures were performed in compliance with the guidelines of the Institutional Animal Care and Use Committees of the Shanghai Ninth People's Hospital (Shanghai, China) and were approved by the Institutional Animal Care and Use Committees of the Shanghai Ninth People's Hospital (Shanghai, China).

Tooth preparation and measurements. Adult (P90) control $(\mathrm{n}=6)$ and Wnt1Cre::iZEG-Dlx2 $(\mathrm{n}=6)$ mice (body weight, 25.2-28.3 g) were sacrificed using $0.8 \%$ pentobarbital sodium via intraperitoneal injection (100 ml/10 g body weight), skinned and eviscerated, then transferred to $95 \%$ ethanol for 2 days. The skulls of mice were then stained with Alcian blue and Alizarin red as previously described (13). The stained teeth were then separated from the alveolar bone under an integrated microscope and were transferred to a solution of $50 \%$ glycerol and $50 \%$ water for imaging.

The root lengths and the ratios of crown/root length in both the maxillary and mandibular first molars in six control and Wnt1Cre::iZEG-Dlx2 mice (twelve teeth respectively) were quantified using digital hand calipers, and all were performed in triplicate.

Micro-computed tomography (CT) scans. The skulls of P90 iZEG-D1x 2 and Wnt1Cre::iZEG-D1x2 mice were collected and fixed with $4 \%$ paraformaldehyde (PFA). Subsequently, micro-CT data were collected using an eXplore Locus MicroCT scanner (GE Healthcare Life Sciences, Milwaukee, WI, USA), using 0.01-mm-thick slices. The 3D reconstructions of the skulls and bone mineral density calculations were completed using GE MicroView software version 2.2 (GE Healthcare Life Sciences).

Histological analysis. Whole heads of embryonic day 13.5 (E13.5) iZEG-Dlx2 and Wnt1Cre::iZEG-Dlx2 mice $(n=6)$ obtained from pregnant mice following anesthetization with $0.8 \%$ pentobarbital sodium via intraperitoneal injection (100 ml/10 g body weight). Jaws of P90 iZEG-Dlx 2 and Wnt1Cre::iZEG-Dlx2 mice $(n=6)$ were obtained following anesthetization with $0.8 \%$ pentobarbital sodium via intraperitoneal injection (100 ml/10 g body weight) and were dissected and fixed in 4\% PFA. P90 jaws were subsequently demineralized in $0.5 \mathrm{M}$ EDTA. The tissues were embedded in paraffin, and $5-\mu \mathrm{m}$ tissue sections were cut, stained with hematoxylin and eosin $(\mathrm{H} \& \mathrm{E})$ and mounted with resinous mounting medium.

Analysis of cell proliferation and apoptosis. A total of 10 mg/ml 5-bromo-20-deoxyuridine (BrdU; Sigma-Aldrich; Merck Millipore, Darmstadt, Germany) solution was injected intraperitoneally at a dose of $100 \mu \mathrm{g} / \mathrm{g}$ body weight into pregnant mice $(n=3)$ at E13.5. The mice were sacrificed by anesthetization with $0.8 \%$ pentobarbital sodium via intraperitoneal injecion (100 ml/10 g body weight) $2 \mathrm{~h}$ after BrdU injection and the embryos were fixed using 4\% PFA, embedded in paraffin and cut into 5 - $\mu \mathrm{m}$-thick sections. Subsequently, BrdU-labeled cells were detected using immunofluorescence staining. Terminal deoxynucleotidyl-transferase-mediated dUTP nick end labeling (TUNEL) assays were used to determine apoptosis in the E13.5 mice and the FITC In Situ Cell Death Detection kit (KeyGen Biotech Co., Ltd, Nanjing, China) was used according to the manufacturer's protocol.

Immunohistochemistry. Heads (E10.5) of iZEG-Dlx2 and Wnt1Cre::iZEG-Dlx2 mice $(n=6)$ obtained from pregnant mice, and jaws of P90 iZEG-Dlx2 and Wnt1Cre::iZEG-Dlx2 mice $(n=6)$ were obtained after sacrifice with $0.8 \%$ pentobarbital sodium via intraperitoneal injection $(100 \mathrm{ml} / 10 \mathrm{~g}$ body weight), following sacrifice with $0.8 \%$ pentobarbital sodium via intraperitoneal injection $(100 \mathrm{ml} / 10 \mathrm{~g}$ body weight and were embedded in paraffin and sectioned at a thickness of $5 \mu \mathrm{m}$. A bone antigen restoration liquid kit (Sunteam Biotech, Shanghai, China) was used for antigen retrieval. Slides were then washed with PBS and blocked for 60 min with $3 \%$ bovine serum albumin (Gibco; Thermo Fisher Scientific, Inc., Waltham, MA, USA) in PBS containing $0.2 \%$ Triton X-100. The sections were subsequently incubated with primary antibodies for anti-Dlx2 (1:100; cat. no. ab18188; Abcam, Cambridge, UK), anti-osteopontin (1:100; cat. no. ab8448; Abcam), anti-BrdU (1:200; cat. no. ab6326; Abcam), anti-Msx2 (1:150; cat. no. ab69058; Abcam), anti-SMAD family member 4 (Smad4; 1:100; cat. no. BS2050; Bioworld Technology, Inc., St. Louis Park, MN, USA), sex determining region Y-box 9 (Sox9; 1:100; cat. no. BS1597; Bioworld Technology, Inc.), anti-transforming growth factor $\beta$ receptor 1 (TGF $\beta R 1) / T G F \beta R 2$ (1:100; cat. nos. BS3257 and BS1696, respectively; Bioworld Technology, Inc.) or anti-runt related transcription factor 2 (Runx 2; 1:100; cat. no. MAB2006; R\&D Systems, Inc., Minneapolis, MN, USA) overnight at $4^{\circ} \mathrm{C}$. The donkey anti-rabbit AlexaFluor 488 and donkey anti-rat AlexaFluor 568 (cat. nos. A21206 and A11077, respectively; Jackson ImmunoResearch Laboratories, Inc., West Grove, PA, USA) secondary antibodies were diluted at 1:300 and incubated with sections for $60 \mathrm{~min}$ at room temperature. Finally, slides were mounted with Vectashield mounting medium containing DAPI (Invitrogen; Thermo Fisher Scientific, Inc.) and images were captured using a fluorescence microscope.

Statistical analysis. Data are presented as the mean \pm standard deviation. The differences between experimental and control 

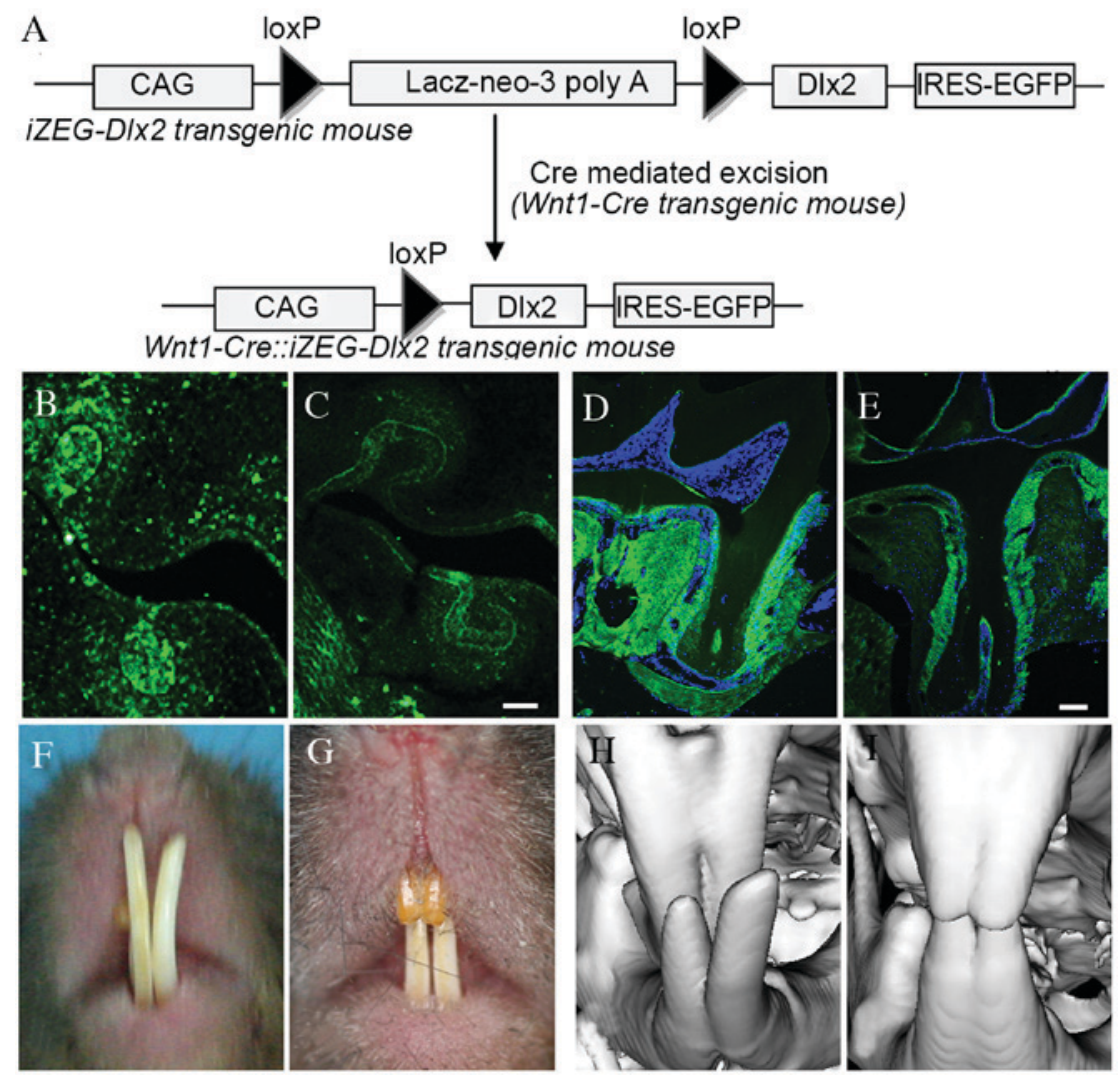

Figure 1. Overexpression of Dlx2 in the teeth of Wnt1-Cre::iZEG-Dlx2 double transgenic mice. (A) Schematic representation of the Cre-mediated excision of the LacZ-Neo-STOP cassette in Wnt1-Cre::iZEG-Dlx2 mice when iZEG-Dlx2 mice were crossed with Wnt1-Cre mice. Immunofluorescence demonstrated that Dlx2 expression was upregulated in the tooth region of (B and C) E13.5 Wnt1-Cre::iZEG-Dlx2 embryos (scale bar, $50 \mu \mathrm{m}$ ) and (D and E) P90 adult mice (scale bar, $100 \mu \mathrm{m}$ ). (F and G) General observation and (H and I) micro-computed tomography examination results showed incisor over-cross bite in P90 Wnt1-Cre::iZEG-Dlx2 mice. Cre, cre recombinase; Dlx2, distal-less homeobox 2; EGFP, enhanced green fluorescent protein; iZEG-Dlx2, transgenic mice conditionally overexpressing Dlx2; Wnt1-Cre::iZEG-Dlx2, double transgenic iZEG-Dlx2 mice expressing Wnt1-Cre; E13.5, embryonic day 13.5.

groups were compared with an independent Student's $t$-test using SPSS version 18.0 (SPSS, Inc., Chicago, IL, USA). $\mathrm{P}<0.05$ was considered to indicate a statistically significant difference.

\section{Results}

Overexpression of Dlx2 in the teeth of Wnt1-Cre::iZEG-Dlx2 double transgenic mice. A schematic diagram of the Wnt1-Cre::iZEG-Dlx2 transgene is presented in Fig. 1A, and the mice were genotyped via PCR using primers specific for EGFP and Cre. Cre-mediated NCC-specific recombination was also detected by PCR analysis using a pair of primers spanning the CAG promoter and the Dlx2 coding sequence (CAG-Dlx2 primers), as previously described (13). Immunofluorescence confirmed that Dlx2 expression was higher in the NCCs derivative tissues, including periodontal tissue, cementum and alveolar bone in embryonic and adult Wnt1Cre::iZEG-Dlx2 mice (Fig. 1B-E) when compared with iZEG-Dlx2 control mice, whereas Dlx2 expression exhibited similar level in others tissues, including long bone and liver, between the Wnt1Cre::iZEG-Dlx2 mice and iZEG-Dlx2 control mice.

Overexpression of Dlx2 leads to tooth dysmorphia. $\mathrm{P} 90$ Wnt1Cre::iZEG-Dlx2 mice exhibited cross-bite malocclusion
(Fig. 1F-I). General observation under an integrated microscope demonstrated that the mandibular and maxillary teeth exhibited shortened root lengths and root morphology dysmorphia (Fig. 2A-H). Measurements of teeth from Wnt1Cre::iZEG-Dlx2 mice and iZEG-Dlx2 mice revealed that root length was significantly reduced $(\mathrm{P}<0.01$; Fig. 2I and $\mathrm{J})$ and the ratios of crown/root length were significantly increased $(\mathrm{P}<0.01$; Fig. $2 \mathrm{~K}$ and $\mathrm{L})$ in both the maxillary and mandibular first molars in Wnt1Cre::iZEG-Dlx2 mice compared with iZEG-Dlx2 mice.

Overexpression of Dlx2 leads to increased cementum deposition and osteoporotic alveolar bone. The present study performed detailed analyses of the maxillary first molar of Wnt1Cre::iZEG-Dlx2 mice at P90. H\&E staining of sagittal sections revealed bone loss and bone defects in the interdental septum and root furcation region in alveolar bone compared with control iZEG-Dlx2 mice (Fig. 3A-H). Notably, increased acellular cementum deposition in Wnt1Cre::iZEG-Dlx2 mice was observed and the molars had sparse and disorganized PDL compared with iZEG-Dlx2 mice. Although the alveolar bone-PDL attachments were defective in Wnt1Cre::iZEG-Dlx2 molars, the cementum-PDL attachments had a normal appearance (Fig. 3E and F). Immunofluorescence results confirmed that the deposition of osteopontin, an osteogenic marker, on the alveolar bone and periodontal tissues was reduced in 


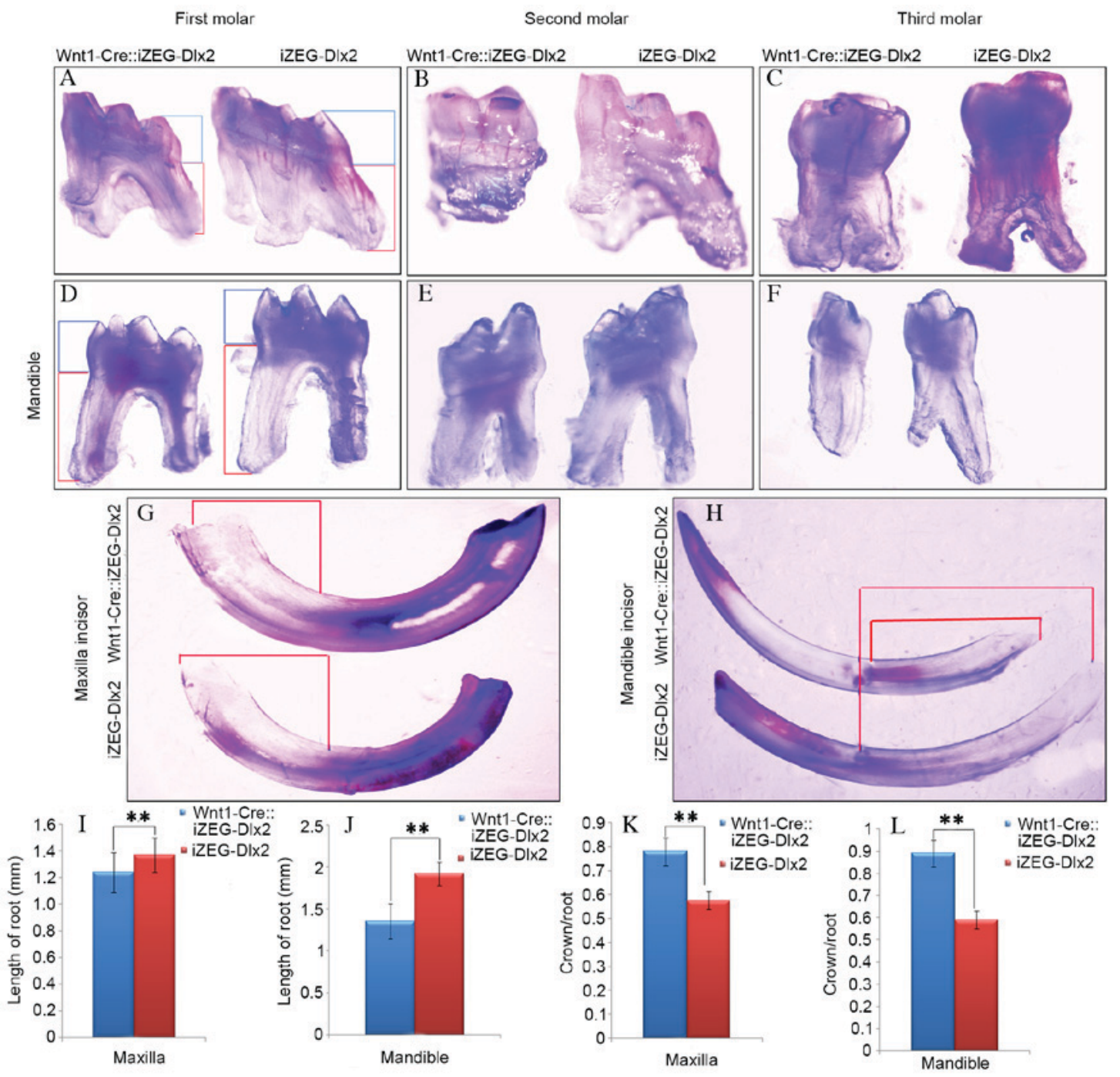

Figure 2. Overexpression of Dlx2 led to tooth dysmorphia. General observation under an integrated microscope revealed shorter roots and root morphology dysmorphia in Wnt1-Cre::iZEG-Dlx2 mice compared with iZEG-Dlx2 mice. The maxillary (A) first, (B) second and (C) third molars, the mandibular (D) first, (E) second and $(\mathrm{F})$ third molars, and the $(\mathrm{G})$ maxillary and $(\mathrm{H})$ mandibular incisors were observed. The red lines indicate the crown lengths and the blue lines root lengths. The root lengths of (I) maxillary and (J) mandibular first molars were significantly reduced in Wnt1-Cre::iZEG-Dlx2 mice compared with iZEG-Dlx2 mice. The (K) maxillary and (L) mandibular crown/root lengths were increased in Wnt1-Cre::iZEG-Dlx2 mice compared with iZEG-Dlx2 mice. ${ }^{* *} \mathrm{P}<0.01$. Dlx2, distal-less homeobox 2; iZEG-Dlx2, transgenic mice conditionally overexpressing Dlx2; Wnt1-Cre::iZEG-Dlx2, double transgenic iZEG-Dlx2 mice expressing Wnt1-Cre.

Wnt1Cre::iZEG-Dlx 2 mice compared with iZEG-Dlx2 mice (Fig. 3I and J). The 3D reconstructed images from micro-CT analysis at P90 also confirmed these results (Fig. 4A-F). At P90, tissue mineral density (TMD), trabecular thickness 3D (Tb.Th 3D) and structure model index (SMI) were quantified using micro-CT examination, which indicated a significant reduction in TMD and Tb.Th $3 \mathrm{D}(\mathrm{P}<0.05$; Fig. $4 \mathrm{G}$ and $\mathrm{H})$, and a significant increase in SMI $(\mathrm{P}<0.05$; Fig. $4 \mathrm{I})$ in the surrounding alveolar bone of Wnt1Cre::iZEG-Dlx2 mice compared with iZEG-Dlx 2 mice (Fig. 4), which clearly demonstrated the osteoporosis of the alveolar bone tissue.

Reduced cellular proliferation and increased apoptosis within the dental germ and alveolar bone of E13.5 Wnt1Cre::iZEG-Dlx2 embryos. A marked decrease in cellular proliferation was detected using BrdU-labeling of the dental germ and alveolar bone in E13.5 Wnt1Cre::iZEG-Dlx2 embryos (Fig. 5A and B). TUNEL assays revealed increased apoptosis in the dental germ and alveolar bone in E13.5 Wnt1Cre::iZEG-Dlx2 embryos compared with iZEG-D1x2 embryos (Fig. 5C and D).

Expression of genes associated with tooth development in Wnt1Cre::iZEG-Dlx2 mice. To identify whether the expression of certain genes that are involved in tooth development was altered by Dlx 2 overexpression, the present study used immunofluorescence staining to detect the expression levels of TGF $\beta R 1$, TGF $\beta R 2$, Smad4, Msx2, Sox9 and Runx2. The expression levels of TGF $\beta R 1$, TGF $\beta$ R2, Smad4 and Msx2, which have previously been demonstrated to contribute to tooth development, were upregulated in the dental germ of E13.5 Wnt1Cre::iZEG-Dlx2 embryos (Fig. 6A-H). Msx2 was also upregulated in the epithelium (Fig. 6G and H). A previous study revealed that a null mutation of Dlx 2 may lead to the reprogramming of odontogenic cells to become chondrogenic and express Sox9 (17). The present study revealed that 

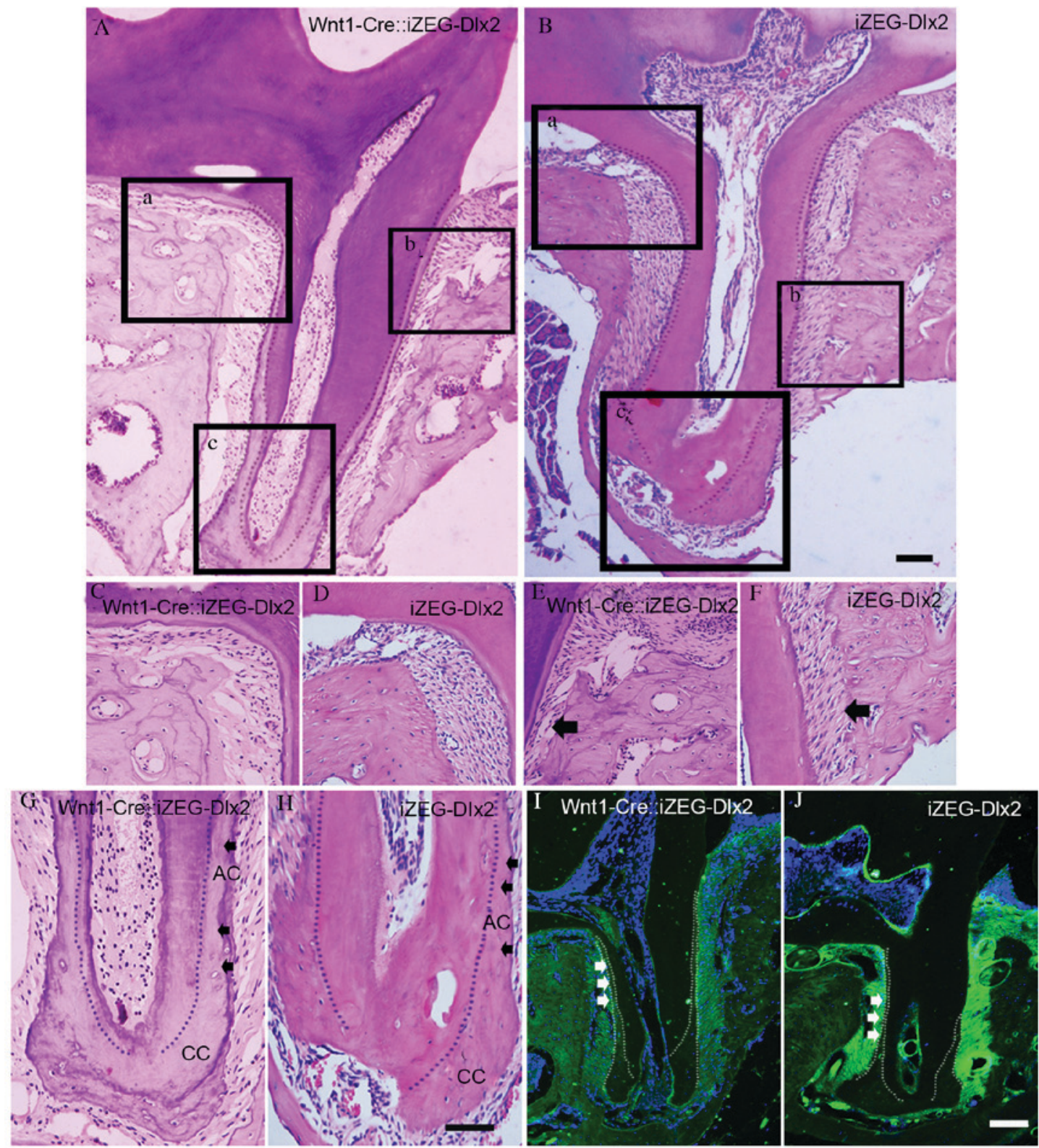

Figure 3. Overexpression of Dlx2 led to increased deposition of cementum and osteoporotic alveolar bone. Hematoxylin and eosin staining of sagittal sections revealed osteoporosis in alveolar bone tissue, increased acellular cementum deposition and defective alveolar bone-PDL attachment in the maxillary first molars of (A) P90 adult Wnt1-Cre::iZEG-Dlx2 mice and (B) iZEG-Dlx2 mice (Scale bars, $200 \mu \mathrm{m}$ ). Bone resorption in the root furcation region in alveolar bone of (C) P90 adult Wnt1-Cre::iZEG-Dlx2 mice and (D) iZEG-Dlx2 mice. Bone resorption and defective alveolar bone-PDL attachment (arrow) in the interdental septum of (E) P90 Wnt1-Cre::iZEG-Dlx2 mice and (F) iZEG-Dlx2 mice. Increased acellular cementum and normal cellular cementum deposition (arrow) in the maxillary first molars of (G) Wnt1-Cre::iZEG-Dlx2 mice and (H) iZEG-Dlx2 mice (Scale bars, $50 \mu \mathrm{m})$. The areas presented in (C-H) are higher magnification images corresponding to the boxed regions in $\mathrm{A}(\mathrm{a}, \mathrm{b}, \mathrm{c})$ and $\mathrm{B}(\mathrm{a}, \mathrm{b}, \mathrm{c})$, respectively. Immunofluorescence staining revealed the decreased deposition of osteopontin on the alveolar bone and periodontal tissues of (I) Wnt1-Cre::iZEG-Dlx2 mice and (J) iZEG-Dlx2 mice (Scale bars, $100 \mu \mathrm{m}$ ). PDL, periodontal ligament, AC, acellular cementum; CC, cellular cementum; Dlx2, distal-less homeobox 2; iZEG-Dlx2, transgenic mice conditionally overexpressing Dlx2; Wnt1-Cre::iZEG-Dlx2, double transgenic iZEG-Dlx2 mice expressing Wnt1-Cre.

Dlx2-overexpression may also increase Sox 9 expression in the dental germ of E13.5 Wnt1Cre::iZEG-Dlx2 mice (Fig. 6I and $\mathrm{J}$ ). By contrast, the expression of Runx2, an osteogenic and odontogenic marker, was downregulated in the dental germ and alveolar bone of E13.5 Wnt1Cre::iZEG-Dlx2 mice compared with iZEG-Dlx2 (Fig. 6K and L).

\section{Discussion}

To the best of our knowledge the present study is the first to describe the effects of Dlx2 overexpression on the formation of dental and periodontal tissues. The current findings suggest that the Dlx2 may be involved in the control of cementum formation. Previous studies revealed that during root cementum formation, cementoblasts exhibited similar characteristics of bone formation to osteoblasts and contribute to matrix deposition in cooperation with periodontal ligament cells $(22,23)$. Molar roots contain two types of cementum: The acellular cementum that is initially deposited, and the cellular cementum that progressively increases in thickness towards the root apex. By contrast, continuously erupted incisors only have acellular cementum (22). A previous study demonstrated 

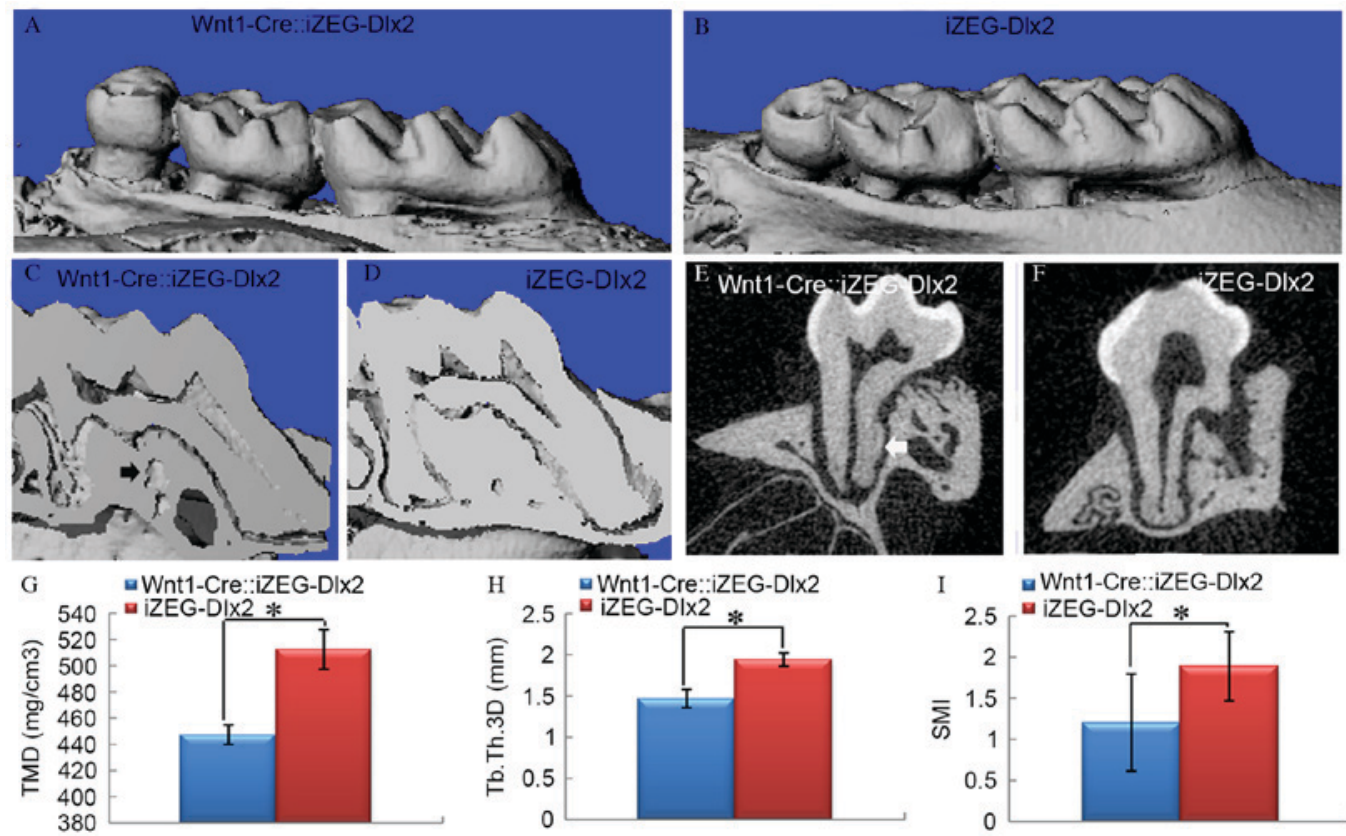

Figure 4. Micro-CT examination. 3D images of alveolar bone absorption (arrow) in (A) P90 Wnt1-Cre::iZEG-Dlx2 mice and (B) iZEG-Dlx2 mice. Sagittal sections of 3D images revealed alveolar bone absorption (arrow) in (C) P90 Wnt1-Cre::iZEG-Dlx2 mice and (D) iZEG-Dlx2 mice. 2D images of increased cementum deposition (arrow) in (E) P90 adult Wnt1-Cre::iZEG-Dlx2 mice and (F) iZEG-Dlx2 mice. (G) TMD, (H) Tb.Th 3D and (I) SMI analyses based on micro-CT examination revealed a reduction in TMD and Tb.Th 3D, and an increase in SMI in the maxillary alveolar bones of P90 Wnt1-Cre::iZEG-Dlx2 mice. " $\mathrm{P}<0.05$. micro-CT, micro-computed tomography; Dlx2, distal-less homeobox 2; iZEG-Dlx2, transgenic mice conditionally overexpressing Dlx2; Wnt1-Cre::iZEG-Dlx2, double transgenic iZEG-Dlx2 mice expressing Wnt1-Cre; TMD, bone mineral density; Tb.Th 3D, trabecular thickness 3D; SMI, structure model index.

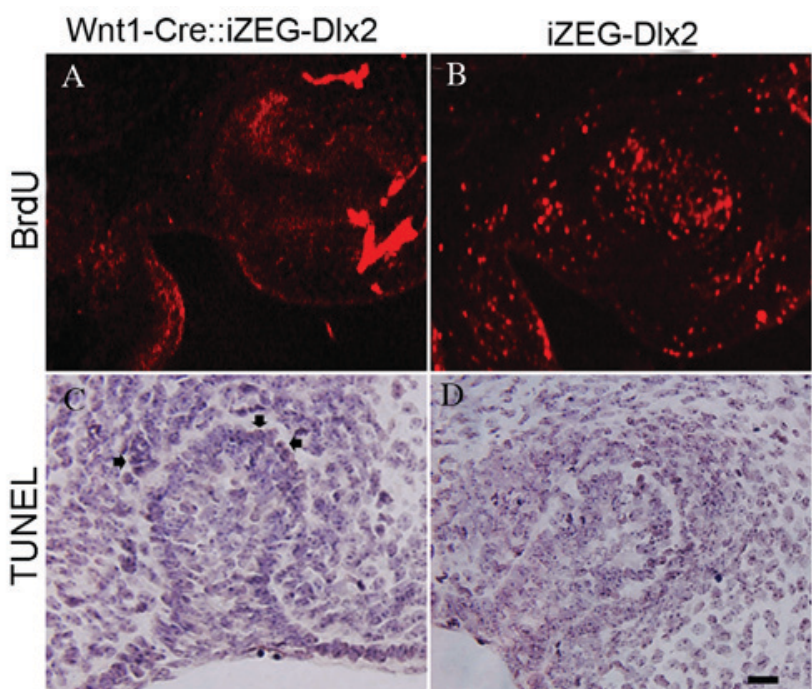

Figure 5. Cell proliferation and apoptosis in the teeth of E13.5 Wnt1-Cre::iZEG-Dlx2 mice. Immunofluorescence staining revealed that there are fewer BrdU-positive cells in the teeth and alveolar bone regions of (A) E13.5 Wnt1-Cre::iZEG-Dlx2 embryos compared with (B) iZEG-Dlx2. TUNEL assays revealed an increase in apoptotic cells (arrows) in the teeth and alveolar bone regions of (C) E13.5 Wnt1-Cre::iZEG-Dlx2 embryos compared with (D) iZEG-Dlx 2 embryos. Scale bars, $50 \mu \mathrm{m}$. BrdU, 5-bromo-20-deoxyuridine; TUNEL, terminal deoxynucleotidyl-transferase-mediated dUTP nick end labeling; E13.5, embryonic day 13.5; Dlx2, distal-less homeobox 2; iZEG-Dlx2, transgenic mice conditionally overexpressing Dlx2; Wnt1-Cre::iZEG-Dlx2, double transgenic iZEG-Dlx2 mice expressing Wnt1-Cre.

that Dlx2 may be continuously present in the epithelial root sheath, from tooth initiation and morphogenesis to the last stages of dental tissue formation and implied that Dlx2 may be involved in the control of cementogenesis (19). The present study determined that Dlx 2 overexpression may result in an increased deposition of acellular cementum, indicating that Dlx2 is crucial for the regulation of cementum formation.

Dlx1 and Dlx2 were the first genes to be identified to contribute to odontogenic patterning and their null mutations solely disrupt the development of the maxillary molar, indicating that there are distinct genetic pathways directing the development of different teeth $(17,24)$. However, the present study revealed that the overexpression of Dlx2 disrupted cementogenesis in maxillary and mandibular molars and incisors. It is possible that the expression of other Dlx genes, such as Dlx5 and Dlx1 may compensate for the absence of Dlx2; however, however that cannot inhibit the effects of Dlx2 overexpression. Unlike cementum deposition, Dlx 2 overexpression may lead to osteoporotic alveolar bone and downregulation of Runx 2 expression, which is consistent with our previous study, which determined that Dlx2 overexpression may impair craniofacial bone development (13). One potential explanation for this discrepancy is that alveolar bone and cementum develop via different molecular mechanisms.

Previous studies have revealed that the development of maxillary molars requires the regional specification of a population of CNCCs by Dlx1 and Dlx2, indicating that the ectoderm mesenchyme may influence tooth morphogenesis $(17,24)$. In the current study, the overexpression of Dlx2 in the neural crest led to disrupted tooth development, confirming this model. Notably, the present study identified that the overexpression of Dlx2 resulted in an ectopic patch of Sox9-expressing cells in the dental germ, whereas a previous study revealed that a null mutation of Dlx2 may also reprogram 

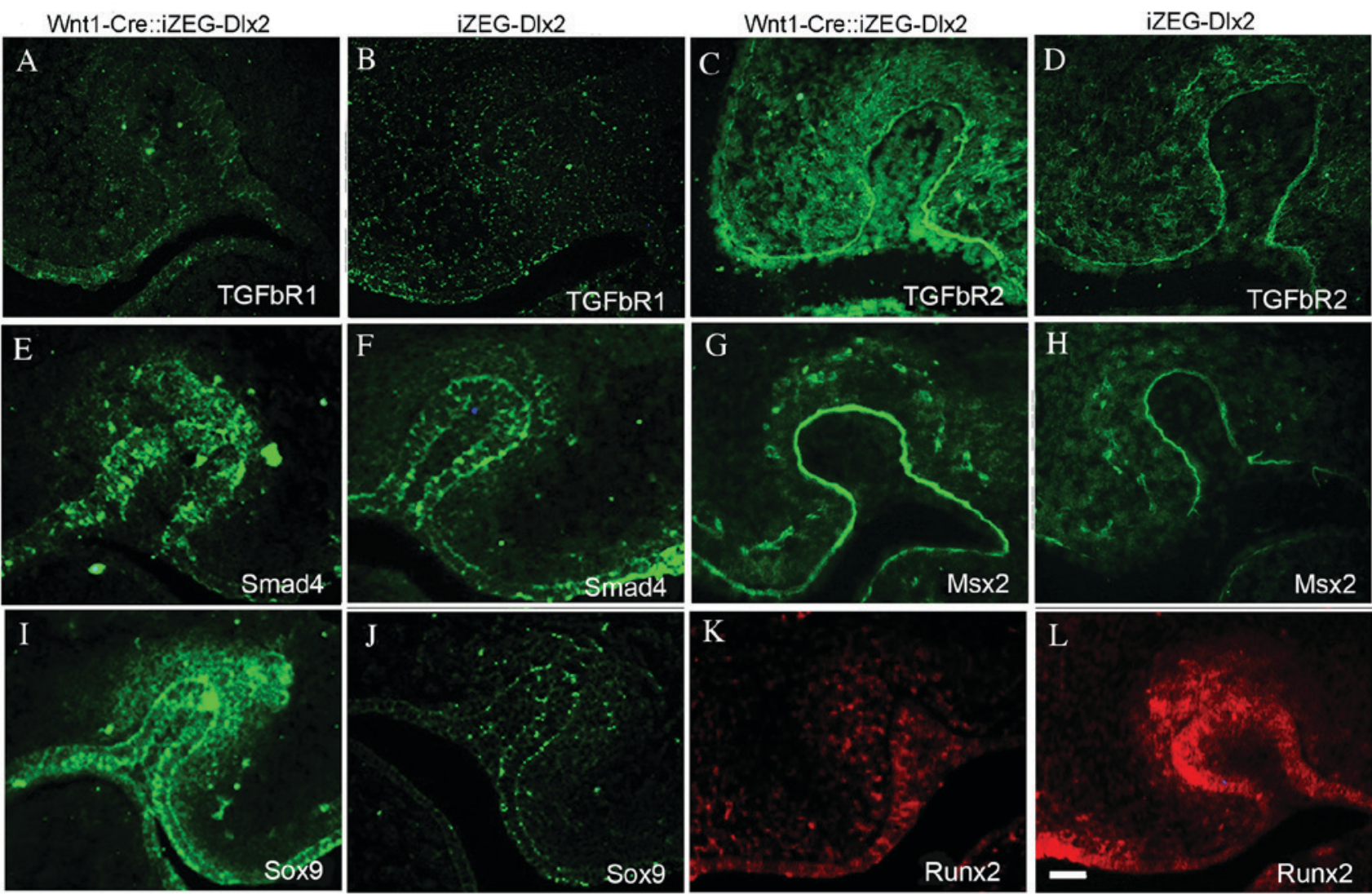

Figure 6. Expression of genes implicated in tooth development in Wnt1Cre::iZEG-Dlx 2 mice. Immunofluorescence staining demonstrated enhanced TGF $\beta R 1$ protein expression in the tooth region of (A) E13.5 Wnt1Cre::iZEG-Dlx2 compared with (B) iZEG-Dlx2 embryos. TGF $\beta$ R2 protein expression is significantly upregulated in the tooth region of (C) E13.5 Wnt1Cre::iZEG-Dlx2 compared with (D) iZEG-Dlx2 embryos. Smad4 protein expression was upregulated in the tooth region of (E) E13.5 Wnt1Cre::iZEG-Dlx2 and (F) iZEG-Dlx2 embryos. Msx2 was upregulated in the dental germ, especially in the epithelium of (G) E13.5 Wnt1Cre::iZEG-Dlx2 compared with (H) iZEG-Dlx2 embryos. Sox 9 expression was upregulated in the dental germ of (I) E13.5 Wnt1Cre::iZEG-Dlx2 compared with (J) iZEG-Dlx2 embryos. Runx2 expression was downregulated in the dental germ of (K) E13.5 Wnt1Cre::iZEG-Dlx2 compared with (L) iZEG-Dlx2 embryos. Scale bars, $100 \mu \mathrm{m}$. E13.5, embryonic day 13.5; Dlx2, distal-less homeobox 2; Wnt1-Cre::iZEG-Dlx2, double transgenic iZEG-Dlx2 mice expressing Wnt1-Cre; iZEG-Dlx2, transgenic mice conditionally overexpressing Dlx2; TGF $\beta R 1 / 2$, transforming growth factor $\beta$ receptor 1/2; Smad4, SMAD family member 4; Msx2, msh homeobox 2; Sox9, SRY (sex determining region Y)-box 9; Runx2, runt related transcription factor 2.

odontogenic cells to express Sox9 (17). The upregulation and downregulation of Dlx2 expression have similar effects on the expression of Sox9, and the mechanism that leads to these expression patterns remains to be elucidated.

Mice overexpressing Dlx2 exhibited short molar tooth roots, and certain maxillary and mandibular incisors had long crowns and an over-cross bite or curved shape. Potentially, the continuously erupting mouse incisors may have acellular cementum on the lingual root analog, revealing a weaker effect of Dlx2 overexpression when compared with cellular cementum. Additionally, due to the deviation of the maxilla, no natural tooth wear was observed through the consumption of hard foodstuffs and gnawing behavior.

Numerous genes have been reported to be involved in tooth development, including Bmp4, Msx2 and Dlx5, which have also been demonstrated to interact with Dlx2 $(1,25)$. The immunofluorescence results in the present study revealed that Dlx2 overexpression may alter the expression of TGF $\beta \mathrm{R} 1$, TGF $\beta \mathrm{R} 2$, Smad4 and Msx2, the four genes that are crucial for tooth development $(1,26,27)$, in tooth tissue, indicating that Dlx2 may disrupt tooth development by interaction with these genes. However, there are various candidate genes that may interact with Dlx2 to regulate tooth development, the exact molecular associations among these genes remain to be elucidated, and require investigation in the future using bioinformatics analysis and experimental verification of their functions.

In conclusion the present study demonstrated that Dlx2 overexpression in mouse CNCCs resulted in incisor cross-bite, shortened tooth roots, increased deposition of cementum, periodontal ligament disorganization and osteoporotic alveolar bone. Additionally, Dlx 2 overexpression increased the expression levels of odontogenesis-associated genes, including TGF $\beta R 1$, TGF $\beta R 2$, Smad4, Msx2 and Sox9 in tooth regions. Therefore, the present study suggested that Dlx 2 overexpression may alter the alveolar bone, cementum and periodontal ligament phenotypes in mice. It is of note that that the present study was limited in that the dental and periodontal phenotypes were assessed in adult mice, whereas the genetic modification usually occurs during embryogenesis; thus, the primary effects of Dlx2 overexpression may occur early in development. Therefore, it is unclear whether the phenotypes observed were due to developmental defects or postnatal degradation secondary to craniofacial deformity and malnutrition. The exact molecular mechanisms underlying the effects of Dlx2 overexpression on dental and periodontal tissue phenotypes require further investigated in the future. 


\section{Acknowledgements}

The authors thank Professor Rulang Jiang (Division of Developmental Biology and Plastic Surgery, Cincinnati Children's Hospital Medical Center, Cincinnati, OH, USA) for his advice in experimental design and biological techniques, and Professor John L.R. Rubenstein (University of California San Francisco, San Francisco, CA, USA) for providing the pCAGGS-Dlx2 plasmid. The current study was supported by National Nature Science Foundation of China (grant nos. 81300842 and 81271122), Open Foundation of Beijing Key Laboratory of Tooth Regeneration and Function Reconstruction and Program for Innovation Research Team of Shanghai Municipal Education Commission.

\section{References}

1. Saadi I, Das P, Zhao M, Raj L, Ruspita I, Xia Y, Papaioannou VE and Bei M: Msx1 and Tbx2 antagonistically regulate Bmp4 expression during the bud-to-cap stage transition in tooth development. Development 140: 2697-2702, 2013.

2. Jia S, Zhou J, Gao Y, Baek JA, Martin JF, Lan Y and Jiang R: Roles of Bmp4 during tooth morphogenesis and sequential tooth formation. Development 140: 423-432, 2013.

3. Zhang Z, Lan Y, Chai Y and Jiang R: Antagonistic actions of Msx1 and Osr2 pattern mammalian teeth into a single row. Science 323: 1232-1234, 2009.

4. Zhao Z, Stock D, Buchanan A and Weiss K: Expression of Dlx genes during the development of the murine dentition. Dev Genes Evol 210: 270-275, 2000 .

5. Fujimori S, Novak H, Weissenböck M, Jussila M, Gonçalves A, Zeller R, Galloway J, Thesleff I and Hartmann C: Wnt/ß signaling in the dental mesenchyme regulates incisor development by regulating Bmp4. Dev Biol 348: 97-106, 2010.

6. Sasaki H, Muramatsu T, Kwon HJ, Yamamoto H, Hashimoto S, Jung HS and Shimono M: Down-regulated genes in mouse dental papillae and pulp. J Dent Res 89: 679-683, 2010.

7. Ozcelik T, Porteus MH, Rubenstein JL and Francke U: DLX2 (TES1), a homeobox gene of the Distal-less family, assigned to conserved regions on human and mouse chromosomes 2 . Genomics 13: 1157-1161, 1992.

8. Stock DW, Ellies DL, Zhao Z, Ekker M, Ruddle FH and Weiss KM: The evolution of the vertebrate Dlx gene family. Proc Natl Acad Sci USA 93: 10858-10863, 1996.

9. Qiu M, Bulfone A, Ghattas I, Meneses JJ, Christensen L, Sharpe PT, Presley R, Pedersen RA and Rubenstein JL: Role of the Dlx homeobox genes in proximodistal patterning of the branchial arches: Mutations of Dlx-1, Dlx-2, and Dlx-1 and -2 alter morphogenesis of proximal skeletal and soft tissue structures derived from the first and second arches. Dev Biol 185: 165-184, 1997.

10. Eisenstat DD, Liu JK, Mione M, Zhong W, Yu G, Anderson SA, Ghattas I, Puelles L and Rubenstein JL: DLX-1, DLX-2, and DLX-5 expression define distinct stages of basal forebrain differentiation. J Comp Neurol 414: 217-237, 1999.
11. Depew MJ, Simpson CA, Morasso M and Rubenstein JL: Reassessing the Dlx code: The genetic regulation of branchial arch skeletal pattern and development. J Anat 207: 501-561, 2005.

12. Qiu M, Bulfone A, Martinez S, Meneses JJ, Shimamura K, Pedersen RA and Rubenstein JL: Null mutation of Dlx-2 results in abnormal morphogenesis of proximal first and second branchial arch derivatives and abnormal differentiation in the forebrain. Genes Dev 9: 2523-2538, 1995.

13. Dai J, Kuang Y, Fang B, Gong H, Lu S, Mou Z, Sun H, Dong Y, Lu J, Zhang W, et al: The effect of overexpression of Dlx2 on the migration, proliferation and osteogenic differentiation of cranial neural crest stem cells. Biomaterials 34: 1898-1910, 2013.

14. McKeown SJ, Newgreen DF and Farlie PG: Dlx2 over-expression regulates cell adhesion and mesenchymal condensation in ectomesenchyme. Dev Biol 281: 22-37, 2005.

15. Gordon CT, Brinas IM, Rodda FA, Bendall AJ and Farlie PG: Role of Dlx genes in craniofacial morphogenesis: Dlx2 influences skeletal patterning by inducing ectomesenchymal aggregation in ovo. Evol Dev 12: 459-473, 2010.

16. Jeong J, Cesario J, Zhao Y, Burns L, Westphal $H$ and Rubenstein JL: Cleft palate defect of Dlx1/2-/- mutant mice is caused by lack of vertical outgrowth in the posterior palate. Dev Dyn 241: 1757-1769, 2012.

17. Thomas BL, Tucker AS, Qui M, Ferguson CA, Hardcastle Z, Rubenstein JL and Sharpe PT: Role of Dlx-1 and Dlx-2 genes in patterning of the murine dentition. Development 124: 4811-4818, 1997.

18. Lezot F, Thomas B, Greene SR, Hotton D, Yuan ZA, Castaneda B, Bolaños A, Depew M, Sharpe P, Gibson CW and Berdal A: Physiological implications of DLX homeoproteins in enamel formation. J Cell Physiol 216: 688-697, 2008.

19. Lezot F, Davideau JL, Thomas B, Sharpe P, Forest N and Berdal A: Epithelial Dlx-2 homeogene expression and cementogenesis. J Histochem Cytochem 48: 277-284, 2000.

20. Duverger O, Zah A, Isaac J, Sun HW, Bartels AK, Lian JB, Berdal A, Hwang J and Morasso MI: Neural crest deletion of Dlx3 leads to major dentin defects through down-regulation of Dspp. J Biol Chem 287: 12230-12240, 2012.

21. Chai Y, Jiang X, Ito Y, Bringas P Jr, Han J, Rowitch DH, Soriano P, McMahon AP and Sucov HM: Fate of the mammalian cranial neural crest during tooth and mandibular morphogenesis. Development 127: 1671-1679, 2000.

22. Foster BL, Soenjaya Y, Nociti FH Jr, Holm E, Zerfas PM, Wimer HF, Holdsworth DW, Aubin JE, Hunter GK, Goldberg HA and Somerman MJ: Deficiency in acellular cementum and periodontal attachment in bsp null mice. J Dent Res 92: 166-172, 2013.

23. Fong H, Chu EY, Tompkins KA, Foster BL, Sitara D, Lanske B and Somerman MJ: Aberrant cementum phenotype associated with the hypophosphatemic hyp mouse. J Periodontol 80: 1348-1354, 2009.

24. Thomas BL and Sharpe PT: Patterning of the murine dentition by homeobox genes. Eur J Oral Sci 106 (Suppl 1): S48-S54, 1998

25. Thomas BL, Liu JK, Rubenstein JL and Sharpe PT: Independent regulation of Dlx2 expression in the epithelium and mesenchyme of the first branchial arch. Development 127: 217-224, 2000.

26. Zhao H, Oka K, Bringas P, Kaartinen V and Chai Y: TGF-beta type I receptor Alk5 regulates tooth initiation and mandible patterning in a type II receptor-independent manner. Dev Biol 320: 19-29, 2008.

27. Huang XF and Chai Y: Molecular regulatory mechanism of tooth root development. Int J Oral Sci 4: 177-181, 2012. 